\title{
A cellular automaton model for the magnetic activity in accretion discs
}

\author{
V. Pavlidou ${ }^{1,2}$, J. Kuijpers ${ }^{3,4}$, L. Vlahos ${ }^{2}$, and H. Isliker ${ }^{2}$ \\ 1 Department of Astronomy, University of Illinois at Urbana-Champaign, Urbana, IL 61801, USA \\ e-mail: pavlidou@astro.uiuc.edu \\ 2 Section of Astrophysics, Astronomy and Mechanics, Department of Physics, University of Thessaloniki, \\ 54006 Thessaloniki, Greece \\ e-mail: vlahos@astro.auth.gr; isliker@astro.auth.gr \\ 3 Astronomical Institute, Utrecht University, PO Box 80 000, 3508 TA Utrecht, The Netherlands \\ 4 Department of Astrophysics, University of Nijmegen, PO Box 9010, 6500 GL Nijmegen, The Netherlands \\ e-mail: kuijpers@astro.kun.nl
}

Received 21 May 1999 / Accepted 15 February 2001

\begin{abstract}
In this paper we attempt, for the first time, to simulate the magnetic activity of an accretion disc using a probabilistic cellular automaton model. Our model is based on three free parameters, the probabilities of spontaneous and stimulated generation of magnetic flux above the surface of the disc $\left(S_{0}\right.$, and, respectively, $P)$, and the probability of diffusive disappearance of flux below the surface $(D)$. The model describes a changing collection of flux tubes which stick out of the disc and are anchored inside the disc at their foot-points. Magnetic flux tubes transfer angular momentum outwards at a rate which is analytically estimated for each single loop. Our model monitors the dynamic evolution of both the distribution of magnetic loops and the mass transfer which results from angular momentum transport due to this distribution. The energy release due to magnetic flaring is also recorded as a function of time and exhibits temporal fluctuations with power spectra that depend on the assumed emission-profile of single flaring loops: (i) for instantaneous emission, the power-spectra are flat at low frequencies and turn over at high frequencies to a power-law with index -0.3 ; (ii) for emission-profiles in the form of one-sided exponentials, the power-spectra exhibit clear power-law behaviour with index -1.7 . Fluctuations with a power law index between -1 and -1.7 are observed in many systems undergoing accretion. We found that our approach allows steady accretion in a disc by the action of coronal magnetic flux tubes alone. If we express the effective viscosity caused by coronal loops in the usual Shakura-Sunyaev $\alpha$ parameter of viscosity, we find values which are in good agreement with observed values.
\end{abstract}

Key words. accretion disks - magnetic fields - methods: numerical - stars: flare - stars: neutron - galaxies: active

\section{Introduction}

An accretion disc is a depot of angular momentum, formed around central objects undergoing mass accretion. Whenever the accreting material has a sufficient amount of angular momentum, this must be disposed of before matter can collapse onto the central compact object. Outward transport of angular momentum by viscous forces takes place on a time-scale which is typically much longer than both the time-scale of radiative cooling and the orbital time-scale. As a result, matter resides at the orbits of lowest energy for given angular momentum and the fluid motion is organized in a series of nearly circular orbits, spiraling slowly inwards (Pringle 1981). Observations suggest that the effective viscosity required to account for

Send offprint requests to: J. Kuijpers,

e-mail: kuijpers@astro.kun.nl the inferred inward radial velocity exceeds the "molecular" viscosity by a factor of $\sim 10^{6}$ (Frank et al. 1992). The nature of this anomalous viscosity has not yet been clarified, although a number of mechanisms have been suggested which, in principle, could provide the required magnitude for the viscosity coefficient $\nu$ (Papaloizou \& Lin 1995; Lin \& Papaloizou 1996). Proposed mechanisms are shocks (Michel 1984; Rozyczka \& Spruit 1993) and waves (Papaloizou \& Pringle 1977; Tagger et al. 1990; Lubow \& Pringle 1993; Stehle \& Spruit 1999), magnetized winds (Blandford \& Payne 1982; Contopoulos \& Lovelace 1994; Mestel 1999), fluid turbulence (Shakura \& Sunyaev 1973), 2-D eddies (Abramowicz et al. 1992; Bracco et al. 2000; Nauta et al. 2000), magnetic turbulence (Lynden-Bell 1969; Shakura \& Sunyaev 1973; Schramkowski \& Torkelsson 1996; Hawley \& Balbus 1999), and coronal magnetic loops (Burm \& Kuperus 1988; 
Heyvaerts \& Priest 1989; Aly \& Kuijpers 1990; Kuijpers 1995). Here we investigate the action of coronal magnetic loops anchored in a disc and its potential significance in providing both the required anomalous viscosity and the observed X-ray variability.

Various observations of accretion discs, both in Active Galactic Nuclei (AGN) and in galactic binary systems, suggest the existence of magnetic flaring phenomena similar to solar flares (Galeev et al. 1979; Pozdnyakov et al. 1983; Nelson \& Spencer 1988; Field \& Rogers 1993; Horne 1994; Fender \& Hendry 2000). A magnetic flare is the violent release of magnetic energy stored in coronal flux tubes by reconnection of magnetic fields. As in the solar case, accretion disc flares are assumed to occur in a magnetically dominated corona, extending on both sides of the disc, as soon as continued energy transfer from the disc into the corona leads to thin magnetic structures along which magnetic reconnection occurs.

The existence of coronae hosting magnetic loops in accretion discs is supported theoretically, on one hand by the Balbus-Hawley instability in Keplerian discs in which seed magnetic fields become amplified (Balbus \& Hawley 1991), and on the other hand by the process of buoyancy of magnetic fields inside discs (Coroniti 1981; Miller \& Stone 1999).

When a coronal magnetic loop anchored in the disc has its foot-points located at different radial distances, it transfers angular momentum from the fast revolving inner gas to the slower outer gas, and either reconnects in a flare event, or else reaches corotation, depending on the size of the loop. It has been suggested that a radially overlapping distribution of magnetic loops could be the origin of the anomalous viscosity appearing in accretion discs (Kuijpers 1995).

Magnetic flaring could also account for the observed variability in X-ray observations of accretion discs. This variability often appears in the form of $1 / f$ fluctuations, a term used to refer to fluctuations having a power spectrum in frequency $f$, exhibiting power law behaviour. In the case of X-ray variability in stellar accretion discs, the power law index has values in the range -1 to -1.7 (Makishima 1988).

Though the extent to which magnetic coronal loops can explain the observed properties of accretion discs is an intriguing question, the global and statistical features cannot be studied by large-scale MHD simulations of interacting magnetic loops. The problem is simply far too complex. It has, however, been shown that a simple analytical model for the power release in magnetic flares in accretion disc coronae in AGN does reproduce the observed temporal properties (de Vries \& Kuijpers 1992; Pounds \& McHardy 1988).

A suggestion for the interpretation of $1 / f$ fluctuations in AGN using an alternative method of study has been made in the work by Mineshige et al. (1994), where a cellular automaton (CA) model generating $1 / f$ fluctuations is presented. In that model, mass is injected in a random fashion into an accretion disc surrounding a black hole.
For each radius from the center of the disc, there is a critical value of the mass density. When the injected mass accumulates and this critical value is exceeded, an unknown instability is invoked and material begins to drift inwards in an avalanche, thereby emitting X-rays. The system evolves towards a self-organized critical state (SOC), with the mass density barely below the critical value in every point of the disc. The authors speculated that the required instability is caused by reconnection of disc magnetic fields.

In our study, we have also made use of a 2-D CA model but not (yet) of a SOC. We study the evolution of a random collection of coronal magnetic loops of finite lengths, assuming only local interactions between neighbouring loops. In this way we are able to simulate the phenomena of magnetic flux emergence, disappearance, flaring and energy release in a magnetized accretion disc corona as well as the angular momentum and mass transport occurring in the disc around a compact object. An important element of our treatment is that we correctly take into account the essentially non-local transport of angular momentum by finite coronal loops correctly.

\section{The model}

\subsection{Physical background}

\subsubsection{The standard model}

Assuming a thin disc $(H(r) \ll r$ where $H$ is the halfthickness of the disc, and $r$ the distance to the central object), in which the pressure is dominated by gas pressure, Shakura \& Sunyaev (1973) have presented a steady, analytical disc solution. The uncertainty in the viscosity mechanism is bypassed by using the "alpha prescription" for the coefficient of kinematic viscosity:

$\nu=\alpha c_{\mathrm{s}} H$

where $c_{\mathrm{s}}$ is the sound speed. Isotropic fluid turbulence is assumed to be the principle source of viscosity in their model, and the "Ansatz" is equivalent to scaling the product of turbulent velocity and eddy size to the local sound speed times the disc half-thickness at a constant ratio, independent of distance. Of course, such a prescription only models the viscosity and leaves open the question of its physical nature, which could well be dominated by magnetic effects, e.g. such as arising from coronal magnetic loops in the present approach.

The thin disc equations are solved in this ShakuraSunyaev approximation for $\Sigma$ (surface density), $H$ (halfthickness), $\rho$ (mass density), $T_{\mathrm{c}}$ (temperature at the central plane of the disc), $\tau$ (optical depth), $\nu$ (viscosity coefficient) and $v_{\mathrm{r}}$ (radial drift velocity) as functions of $M$ (mass of the central compact object), $\dot{M}$ (mass transfer rate), $r$ (distance from the center of the disc) and the Shakura-Sunyaev parameter $\alpha$. 
In the thin disc approximation, the circular fluid velocity $v_{\phi}$ is very close to the Keplerian value. A thin ring of material with radius $r$ is rotating at an angular velocity

$\Omega_{\mathrm{K}}(r)=\sqrt{\frac{G M}{r^{3}}}$.

\subsubsection{Magnetic activity}

Based on the Balbus-Hawley instability (Balbus \& Hawley 1991) Tout \& Pringle (1992) have constructed a disc dynamo. Starting with an initially vertical seed magnetic field, the instability creates a radial field component, which in turn is sheared by differential rotation, and gives rise to an azimuthal component. Buoyancy then acts on this azimuthal component and recreates a vertical component, after which the cycle is repeated.

In our model, we adopt the view that the magnetic field appears in the form of intermittent flux tubes inside the disc dynamo rather than in the form of spatially smooth fields. These flux tubes, while anchored inside the disc, emerge in the disc corona where they form the dominant pressure contribution, and, therefore, are space filling.

Though the details of the interactions between these discrete flux tubes are highly complex, we can sketch a general picture guided by two of the best-studied cases of magnetized objects: the solar magnetic field and the galactic magnetic field. In both cases, observations support the existence of discrete flux tubes.

The life-cycle of flux tubes on the solar surface has been well-studied, using a CA model, by Wentzel \& Seiden (1992) and Seiden \& Wentzel (1996), and we model the main processes of the appearance, disappearance and interaction of magnetic flux tubes in an accretion disc corona in a similar fashion:

Spontaneous generation: Flux tubes embedded in the disc experience a buoyancy force which tends to raise the tubes towards the surface. Provided that the flux tubes have been amplified by the disc dynamo enough to survive multiple fragmentation during their up-welling, they emerge from the disc into the corona in a stochastic fashion. Such a stochastic emergence of flux tubes in regions where a magnetic field is absent (or very weak) has been observed for the Sun (Howard 1996).

Stimulated generation: As is the case on the Sun (Weiss 1997) the emergence of a flux tube from the main body of the disc may lead either to fragmentation of the emerging loop, or to disturbing other submerged flux tubes, or both. In the first case, the fragments of the original loops emerge sequentially and close to each other. In the second case, other submerged flux tubes may be triggered to emerge. In either case, there is an increased probability for the emergence of new loops in the spatial "neighbourhood" of existing loops. The time-scale in which this "stimulated" appearance occurs will be of the order of the rise time of a loop from the center of the disc to its surface. The rise velocity $v_{\text {rise }}$ of the flux tube will be of the order of an effective Alfven speed $v_{\mathrm{A}}$ which is approximately equal to the local sound speed $c_{\mathrm{s}}$ if one requires the magnetic field pressure inside a flux tube to be equal to the ambient gas pressure inside the disc $p_{\text {magnetic }}=p_{\text {gas }}$. Then

$t_{\text {rise }}(r)=\frac{H(r)}{v_{\text {rise }}(r)} \simeq \frac{H(r)}{c_{\mathrm{S}}(r)} \simeq \frac{1}{\Omega_{\mathrm{K}}(r)}$.

Flaring: Solar flares are thought to occur when the largely force-free - electric current system in coronal loops has increased to such an extent that reconnection of the magnetic field sets in impulsively, and releases stored magnetic energy in a violent manner. The increase in electric currents is caused by kinematic distortion of coronal flux tubes due to subphotospheric flows. In the case of accretion discs, shearing and twisting caused by differential rotation of the disc has a similar effect as subphotospheric flows in the Sun, and is expected to cause distortion, subsequent reconnection, and energy release of magnetic fields in the corona of an accretion disc. As it is the Keplerian shear which distorts the magnetic field, its effect varies with the initial orientation of the flux tubes upon emergence. When, at the time of emergence, the loop is oriented along the radial direction, the Keplerian flow will induce an azimuthal field component and, at the same time, a twist inside the tube. Also, the length of the tube will increase. When, initially, the loop is oriented at a large angle with respect to the radial direction, the change in the azimuthal field component will be relatively small, while the main effect of the flow on the tube is to increase its length. The loop becomes merely elongated without much increase in the internal coronal current density. Further, the effect of twisting is determined by the differential rotation frequency of both foot-points, and occurs on a long time scale, as compared to the Keplerian time. Therefore, we neglect the effect of twisting, and assume that an, initially, radial loop is more susceptible to flaring than when it emerges at a non-zero angle. In the extreme case of a loop which emerges at right angles to the radial direction, the loop is not affected in any way by differential rotation.

Diffusive disappearance: On the Sun, coronal flux is known to disappear due to diffusive destruction. In the solar corona, active regions are observed to "fray". First, the magnetic field at the circumference falls below an observable level, and later, the inner parts of the active region follow. Although the details of the disappearance process are unclear, it is believed that the flux constituting the active region undergoes lateral fragmentation and the fragments spread out so that the magnetic field strength is reduced to a non-observable level. Loop fragments subsequently submerge due to forces caused by subphotospheric flows (Howard 1996). In the accretion disc case we shall assume that coronal flux tubes exhibit similar behaviour, with flux concentrations tending to diffuse away towards areas with weak or no magnetic field.

\subsubsection{Angular momentum transfer}

Coronal loops with footpoints lying at different distances from the center of the disc connect areas with different 
specific angular momentum. The loops transport angular momentum effectively from the fast revolving inner regions of the disc to its outer parts (Aly \& Kuijpers 1990). This raises the question of whether a radially overlapping distribution of coronal loops could provide an effective viscosity mechanism which can account (at least partly) for the high accretion rates observed in accretion discs.

Here, we follow the estimates of the rate of transport of angular momentum in an individual coronal loop, as summarized in the Appendix. We distinguish between the cases of a large, flaring loop and a small loop which does not flare. A flaring loop transfers angular momentum effectively during a time

$\tau_{\mathrm{f}}=2 /\left(3 \Omega_{\mathrm{K}}\right)$

(after which it flares), and at a rate

$\dot{J}_{\mathrm{f}}=2 A_{2} \rho c_{\mathrm{s}}^{2} r_{2} f_{\mathrm{c}}^{2}=2 A_{2, \operatorname{phot}} \rho c_{\mathrm{s}}^{2} r_{2} f_{\mathrm{c}}$,

where $r_{2}$ is the distance of the outer foot-point of the loop from the central object, $A_{2}$ is the area covered by the

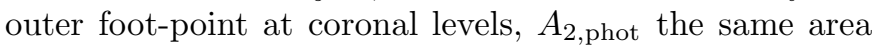
at the photospheric level, $\rho=\rho\left(r_{2}\right)$ is the mass density in the disc, $c_{\mathrm{S}}\left(r_{2}\right)$ is the sound speed in the disc, and $f_{\mathrm{c}}$ is a correction factor which expresses the coronal magnetic field value - which is relevant for the torque - in term of the magnetic field value at the (photospheric) surface $B_{\text {cor }}=f_{\mathrm{c}} B$ (implying $f_{\mathrm{c}} A=A_{\text {phot }}$ ). We define the $l o$ cal surface filling factor $F_{\mathrm{ff}}$ at distance $r$ as the relative area of the ring at distance $r$ covered by magnetic flux. As the magnetic field in the corona dominates over the local gas, it fills the entire corona ("space-filling"), and flux conservation then implies that typically $f_{\mathrm{c}}=F_{\mathrm{ff}}$. To take account, however, of a disc with a few isolated loops (the case of a very small filling factor) we use

$f_{\mathrm{c}}=\max \left\{F_{\mathrm{ff}}, H^{2} / L^{2}\right\}$

on geometrical grounds. Here $L$ is the linear separation of the foot-points of the isolated loop.

A small loop (radial separation $r_{2}-r_{1} \leq 8 H / 9$ ) transfers angular momentum effectively during a time

$\tau_{\mathrm{s}}=\frac{1}{\Omega_{\mathrm{K}}} \cdot \sqrt{\frac{r_{2}-r_{1}}{2 H}}$,

after which its foot-points reach corotation, and at a rate

$\dot{J}_{\mathrm{s}}=3 \frac{\sqrt{r_{2}-r_{1}}}{2^{1.5} \sqrt{H}} \dot{J}_{\mathrm{f}}$

\subsection{The cellular automata approach}

Based on the above processes for the formation and evolution of loops, a CA model is proposed here, which focuses on the global evolution of the magnetic activity of an accretion disc. The main elements of the CA approach are a) Discretization in space and time: interacting elements are positioned on a discrete, usually periodic, lattice and all interactions and events occur at discrete steps; b) "translation" of the complex physical laws which govern the system to a set of simple rules describing the interactions between elements; c) localized interactions: all interactions between elements are assumed to be local and the "sphere of influence" of any single element is constrained within its immediate spatial neighbourhood.

A CA model reproduces global features of the behaviour of a system that cannot be predicted in advance as a direct conclusion of the rules controlling the local interactions but are the result rather of their non-linear synthesis. Such a study allows us to isolate specific sets of microscopic properties which are responsible for specific observed global properties. Conversely, if the CA model reproduces the observed global features successfully, this indicates that the "microscopic" physics, as embedded in the selected rules, characterize the system.

\subsubsection{The Shakura-Sunyaev disc solution}

In our approach, the Shakura-Sunyaev disc solution initializes our computations. Further, it is used to determine the geometric features of our model. It serves as a plausible assumption for the geometry of our automaton and the central temperature and density of the disc.

We restrict ourselves to the region of the disc which is dominated by gas pressure over radiation pressure. The Shakura-Sunyaev solution determines the initial half thickness $H(r)$, central temperature $T_{\mathrm{c}}(r)$, and mass density $\rho(r)$ as a function of the distance $r$ from the center of the disc:

$H(r)=1.7 \times 10^{8} \alpha^{-1 / 10} \dot{M}^{3 / 20} M^{-3 / 8} r^{9 / 8} f_{\mathrm{r}}^{3 / 5} \mathrm{~cm}$,

$\rho(r)=3.1 \times 10^{-8} \alpha^{-7 / 10} \dot{M}^{11 / 20} M^{5 / 8} r^{-15 / 8} f_{\mathrm{r}}^{11 / 5} \mathrm{~g} / \mathrm{cm}^{3},(10)$

$T_{\mathrm{c}}(r)=1.4 \times 10^{4} \alpha^{-1 / 5} \dot{M}^{3 / 10} M^{1 / 4} r^{-3 / 4} f_{\mathrm{r}}^{6 / 5} \mathrm{~K}$,

where $r$ is given in $10^{10} \mathrm{~cm}, M$ in solar masses, $\dot{M}$ in $10^{16} \mathrm{~g} / \mathrm{s}$ and $f_{\mathrm{r}}=\left[1-\left(\frac{R_{*}}{r}\right)^{1 / 2}\right]^{1 / 4}$ where $R_{*}$ is the radius of the compact object (Frank et al. 1992).

Despite the fact that for the initialization of our automaton and for evaluating temperatures we have used, for simplicity, $\alpha=$ const, there is no physical reason why $\alpha$ should be independent of $r$. If, however, $\alpha$ did depend on $r$, the functional dependence of $H, \rho$ and $T_{\mathrm{c}}$ on $r$ would change accordingly.

\subsubsection{Geometry}

We model one side of the disc only, and assume that the events occur symmetrically on both sides of the disc at the same time. We model the disc as a 2-D circular grid consisting of $\sim 300$ rings.

1. Each ring rotates at a Keplerian angular velocity $\Omega_{\mathrm{K}}(r)$ given by Eq. (2);

2. The width of each ring is equal to $8 H(r) / 9$ (see Appendix), where $H(r)$ is the half-thickness of the disc (as given by the Shakura-Sunyaev disc solution), and $r$ the radius of the ring; 


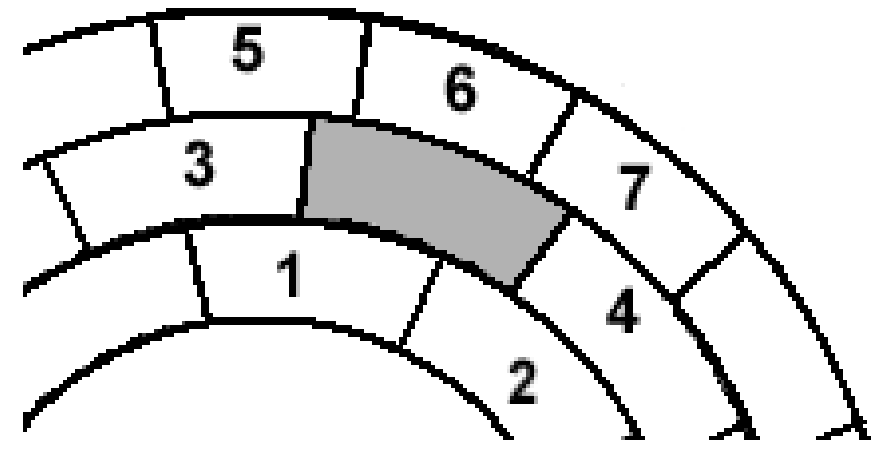

Fig. 1. The nearest neighbours of a cell in our grid. The neighbouring cells are the only grid elements with which a cell is interacting.

3. Each ring is divided into a number of cells, each of which may host a loop foot-point at any time. A cell has one of two possible states: either it hosts a loop foot-point and is completely covered with flux, or it does not host a foot-point and it contains no flux at all;

4. The magnetic field strength at each ring is calculated by putting the magnetic pressure equal to the gas pressure in the disc $p_{\text {gas }}=B^{2} / 8 \pi$ and using the ShakuraSunyaev value for the gas pressure: $p_{\text {gas }}=\frac{\rho k T_{\mathrm{c}}}{\mu \mathrm{m}_{\mathrm{p}}}$ with $\rho$ and $T_{\mathrm{c}}$ given by the relations (10) and, respectively, (11). The magnetic field strength calculated this way corresponds to the interior of the disc and is therefore an upper limit to the magnetic field strength at the surface (which is of interest in our case) rather than its actual value. Still, to retain simplicity in our calculations, we use this value of $B$ for the magnetic flux tubes at the surface;

5. The number of cells in each ring is now adjusted in such a way that each cell corresponds to the same amount of flux, independent of distance from the center. In this way, loops with foot-points lying at different $r$ satisfy conservation of magnetic flux. Thus, we find for the number of cells in each ring

$N(r)=C r^{13 / 16}$,

where $C$ is a constant depending on the amount of flux that each individual cell can host, and is a parameter controlling the spatial resolution of the model;

6. Each cell is in physical contact with a number of cells: in the same ring, in the adjacent outer ring, and in the adjacent inner ring (Fig. 1) Since the number of cells increases as we move outwards, the exact number of adjacent cells depends on the relative orientation of the rings. These cells are the "neighbours" of the cell considered and constitute its "sphere of influence". A loop only interacts with cells neighbouring its foot-points. Since the disc rotates differentially, the neighbours of a cell differ in time in general;

7. Initially, each ring contains an amount of mass which is calculated from the Shakura-Sunyaev value for $\rho$ at the corresponding $r$.
In order to use the Shakura-Sunyaev equations to compute the various geometric features of the model, we need to chose initial, fixed values for $M, \dot{M}$ and $\alpha$. $M$ characterizes the central compact object and in our calculations is equal to $1.4 M_{\odot}$, a typical value for the mass of a neutron star. For $\dot{M}$ and $\alpha$ we use initial values close to the ones observed in actual accreting systems.

The modeled region of the disc extends from approximately 2 to 10 stellar radii.

\subsubsection{Magnetic activity and angular momentum transfer rules}

In line with the previous constraints and physical assumptions, we model our CA as follows:

Initial loading: We initialize the automaton by randomly distributing bipolar loops of various sizes and orientations so that approximately $1 \%$ of the surface is covered by magnetic flux. We then let the loops evolve dynamically according to the following rules:

Spontaneous generation: The stochastic emergence of loops is simulated by ascribing a probability $S$ of spontaneous generation to each cell hosting no magnetic flux. $S$ is the probability for an empty cell to host a loop foot-point in the next time step. The other foot-point of the newly generated loop appears at the same time step at a random position (and, therefore, with various orientations of the loop) within a band of rings of maximum radial separation $\Delta r_{\max } \approx 50 \mathrm{H}$ from the ring hosting the first foot-point.

This probability $S$ is a parameter directly associated with the amount of flux present in a certain region of the disc. We require $S$ to depend on the actual (instead of the steady Shakura-Sunyaev) mass density of each ring. The reason is as follows. As the model evolves, $\rho$ deviates from the value given by the Shakura-Sunyaev disc solution because the mass transfer is governed not by the initially chosen $\alpha$, but by the presence of coronal loops. As mass accumulates at a given radius, new magnetic field, once it appears, is expected to be relatively strong $\left(B \propto \sqrt{p_{\text {gas }}} \propto\right.$ $\sqrt{\rho}$ ). In reality, therefore, the magnetic field at a given radius varies in time, while in our model, for simplicity, we calculate the local magnetic field value from the ShakuraSunyaev solutions for $\rho$ and $T$. In order to take the timedependence of $\rho$ and, consequently, of $B$ into account, we introduce a $\rho$-dependence in $S$ instead, and demand

$S=\min \left[S_{0} \sqrt{\rho / \rho_{0}}, 1\right]$

where $\rho_{0}$ is the Shakura-Sunyaev value of $\rho$ for the ring considered, and $S_{0}$ is the spontaneous generation probability corresponding to a disc having a Shakura-Sunyaev mass distribution. $S_{0}$ is a free parameter of the model and is taken to be space-independent.

Stimulated generation: This process is simulated by allowing existing loops to stimulate the emergence of new loops: cells which, in a certain time step, have no magnetic flux, have an increased probability of hosting a loop footpoint in the next time step if they are neighbouring an 
already existing loop. Both foot-points of the stimulated loop are taken to emerge near the respective foot-points of the stimulating loop.

In general this probability is a second free parameter related to the internal dynamics of the interaction of flux tubes. In this article we assume that it is related to the rise time of the flux tube, and keep its value constant. Since the estimated rise time of a loop (Eq. (3)) is

$t_{\text {rise }}(r)=\frac{1}{\Omega_{\mathrm{K}}(r)}=\sqrt{\frac{r^{3}}{G M}}$

we take for the probability of stimulated generation $P$ per time step

$P=\frac{t_{0}}{t_{\text {rise }}}=\sqrt{\frac{G M}{r^{3}}} t_{0}$,

where $t_{0}$ is arbitrary in general, but, in the present calculations, set equal to our time-step $t_{\mathrm{ts}}$, which we take to be 0.1 times the rotation period of the innermost ring.

Flaring: Loops which, at the time of their birth are too oblique with respect to the radial direction, are expected to be elongated rather than sheared by differential rotation and do not undergo flaring. Of course, they would be twisted on a much longer time scale. To simulate this phenomenon while retaining simplicity, we have adopted a "crude" distinction between loops deviating less than $45^{\circ}$ from the radial direction, which flare, and loops deviating more than $45^{\circ}$ from the radial direction, which do not flare. The latter kind does not transfer angular momentum. To a first approximation, we consider such loops unimportant, and disregard them in the present study.

We now consider loops which, at the time of birth, do not deviate more than $45^{\circ}$ from the radial direction. A loop with a radial foot-point separation greater than $8 H / 9$ (that is to say, greater than the width of one ring) is taken to undergo flaring, after a lifetime equal to $\tau_{\mathrm{f}}$. In our model, after flaring, magnetic field remnants disappear from the grid. Loops with a radial separation less than $8 H / 9$ are assumed not to flare, since they reach corotation before shearing can distort them sufficiently (Kuijpers 1995). After reaching corotation, such "small" loops do not transfer angular momentum and, for simplicity, disappear from the grid.

Diffusive disappearance: For every neighbour free of magnetic flux an existing loop has a probability $D$ of disappearing in the next time step. Thus, the total probability of a loop of diffusing increases with the number of free cells adjacent to its foot-points and is equal to

$D_{\text {total }}=1-(1-D)^{n}$,

where $n$ is the number of free neighbours. $D$ is a free $p a-$ rameter of the model.

Energy release: In our model we can monitor the energy released by coronal flaring activity, and in particular its temporal fluctuations. Of course, with the present assumptions, the magnetic energy release must remain much smaller than the thermal emission for reason of consistency. The energy released in each flare is at least equal to the free energy stored in the magnetic field of the flaring loop. In our model of a force-free corona, the magnetic virial theorem places an upper limit on the free magnetic energy (Aly 1985), which is estimated to be of magnitude (Kuijpers 1992)

$f_{\mathrm{c}}^{2} \frac{B_{z}^{2} L A}{8 \pi}=f_{\mathrm{c}} \frac{B_{z}^{2} L A_{\mathrm{phot}}}{8 \pi}$

where $B_{z}$ is the vertical component of the magnetic field at the surface (photosphere) of the $\operatorname{disc}\left(f_{\mathrm{c}} B_{z}\right.$ is the vertical component in the force free corona just above the disc), $A$ is the foot-point area at coronal levels, and $A_{\text {phot }}$ that at the photosphere, and $L$ is the linear separation between footpoints ( $L A$ is an estimate of the volume of the loop).

Angular momentum and mass transfer: To study the angular momentum transfer by an evolving distribution of coronal loops, we have adopted the following rules to simulate the transport of angular momentum and the accompanying mass flow:

1. A flaring loop transfers angular momentum from the inner to the outer foot-point during a number of time steps given by the integer part of $\tau_{\mathrm{f}} / t_{\mathrm{ts}}$, where $\tau_{\mathrm{f}}$ is the flare time and $t_{\mathrm{ts}}$ is the time interval corresponding to one time step. The rate of angular momentum transfer equals $\dot{J}_{\mathrm{f}}$, given by $(5)$;

2. A small loop (a loop whose radial separation does not exceed $8 H / 9$ ) transfers angular momentum during a number of time steps equal to the integer part of $\tau_{\mathrm{s}} / t_{\mathrm{ts}}$ at a rate $\dot{J}_{\mathrm{s}}$ given by $(8)$;

3. After a number of time steps equal to their lifetime, flaring loops release energy and then disappear while small loops do not release energy and are then taken out. The actual lifetime of a loop can, however, be smaller than its calculated lifetime due to diffusive destruction. In this case, of course, the angular momentum transfer ceases at the same time;

4. When a loop transfers angular momentum of the amount $\Delta J=\dot{J} t_{\mathrm{ts}}$ per time step from the inner to the outer cell, the fluid at the outer foot-point gains this amount $\Delta J$ of angular momentum and thus tends to move outwards. On the other hand, the fluid at the inner foot-point loses the same amount $\Delta J$ of angular momentum and tends to move inwards. We assume that a mass $m_{\mathrm{f}}\left(r_{2}\right)\left(m_{\mathrm{f}}\left(r_{1}\right)\right)$ is transferred outwards (inwards) to the next outer (inner) ring, and stops interacting with the loop. The amount of mass $m_{\mathrm{f}}$ that leaves a cell and goes outwards (inwards), due to an increase (a decrease) of its angular momentum equal to $\Delta J$, can be calculated from the difference between specific angular momenta between adjacent rings:

$\Delta J=\frac{m_{\mathrm{f}} \sqrt{G M}}{2} \frac{\Delta r}{\sqrt{r}}$ 


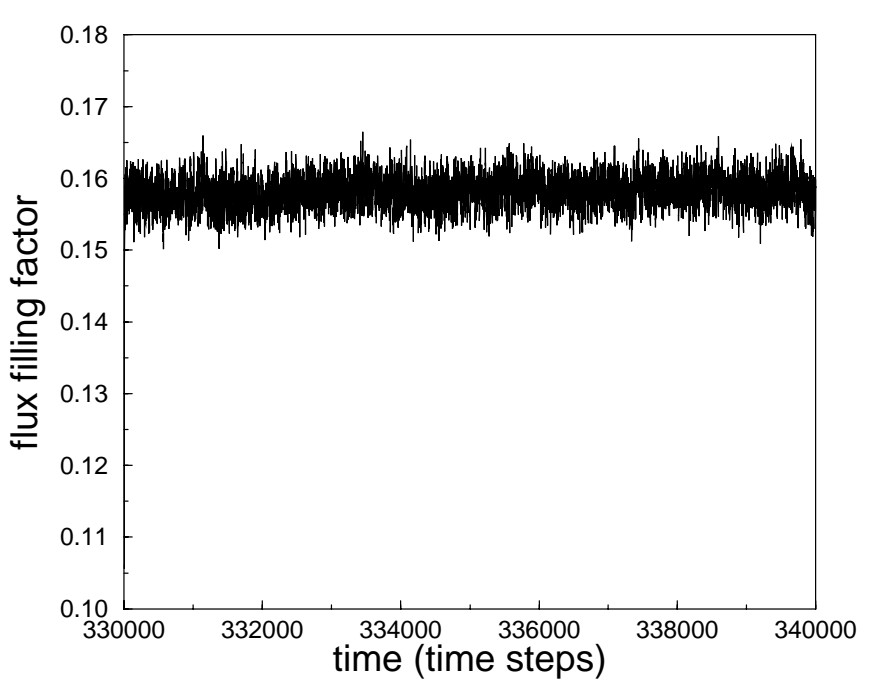

Fig. 2. The flux filling factor $F_{\mathrm{ff}}$ as a function of time $(D=0.1$, $\left.S_{0}=0.05\right)$. Stabilization occurs around the value 0.158 .

where $\Delta r=8 H / 9$ is the width of a ring. Therefore, we have

$m_{\mathrm{f}}=\frac{2 \Delta J}{\sqrt{G M}} \frac{\sqrt{r}}{\Delta r}$,

where $\Delta J$ is the angular momentum transfer in one time step. Since $\Delta r=H(r) 8 / 9 \propto r^{9 / 8}$ and $\Delta J \propto \dot{J} \propto$ $\left(\rho(r) T_{\mathrm{c}}(r)\right)^{0.5} r$, the transferred mass varies with radius as $m_{\mathrm{f}} \propto r^{-15 / 16}$. This implies that the angular momentum transfer in an individual loop causes more mass to slide inwards than to be ejected outwards. This results in a net inward transport of mass, and is a means of verifying the physical validity of our model.

These rules complete our CA model which is now fixed by three physical quantities (mass of central compact object $M$, mass input rate $\dot{M}$, coefficient of viscosity $\alpha$ ) and three free parameters (the probabilities of spontaneous generation $S_{0}$, of stimulated generation (the constant $t_{0}$ in Eq. (15)), and of diffusive disappearance $D$ ). In this article we have only investigated the dependence on $S_{0}$ and $D$ and keep $t_{0}$ constant.

\section{Results}

\subsection{Distribution of coronal magnetic loops}

For each set of $S_{0}$ and $D$ the local flux filling factor of the disc (the fraction of the disc ring that is covered by flux) reaches a stable mean distribution (as a function of radius), about which it exhibits small fluctuations. Time evolution and stabilization of the flux filling factor $F_{\mathrm{ff}}$ for characteristic values of $S_{0}$ and $D$ are shown in Fig. 2.

For all values of $S_{0}$ and $D$ the time evolution is very similar, while the flux filling factor $F_{\mathrm{ff}}$ stabilizes around different mean values.

Coronal flux tubes tend to form organised "active regions", a result of the stimulated generation process. This tendency can be observed in Fig. 3, where a face-on snapshot of a disc with filling factor $f_{\mathrm{c}}=0.158$ is presented.
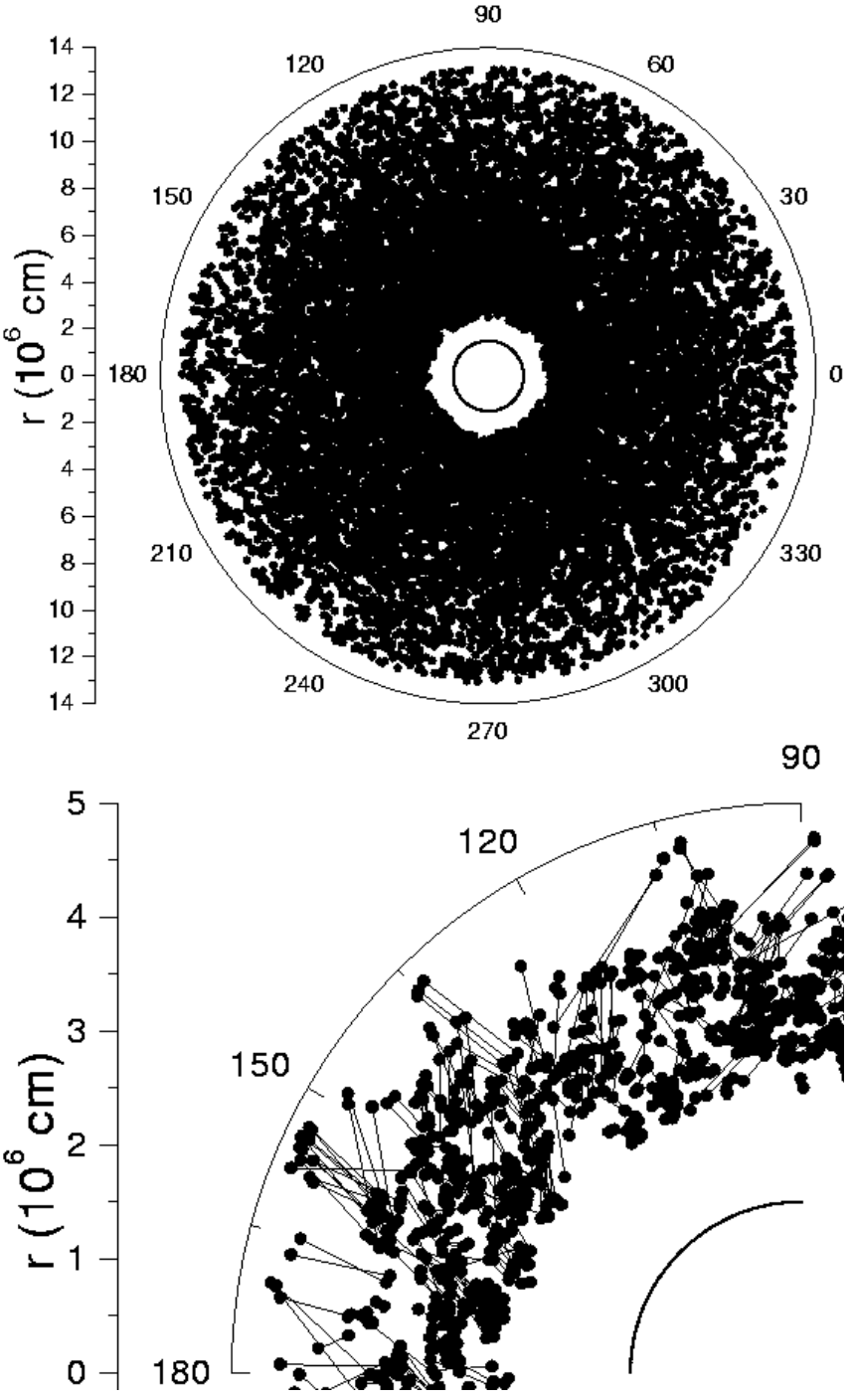

Fig. 3. Top: a face-on snapshot of the modeled region of the disc, where the spatial distribution of the coronal magnetic loops can be seen. The central compact object is also drawn to scale at the center of the accretion disc. Bottom: a magnified section of the picture above, where the active regions can be easily discerned. Again, the compact object is drawn at the center of the disc $\left(D=0.1, S_{0}=0.05\right.$, leading to a steadystate filling factor $\left.f_{\mathrm{c}}=0.158\right)$.

\subsection{Mass transfer and mass distribution}

For a given combination $\left\{S_{0}, D\right\}$ we can determine the value of the mass input rate $\dot{M}$ and the effective viscosity parameter $\alpha$ around which the system stabilizes. In a stabilized disc the total mass fluctuates around a steady mean value. The stable rate of mass transfer is achieved by angular momentum transport solely due to coronal magnetic loops!

We start our computation (at a fixed $M$ equal to $\left.1.4 M_{\odot}\right)$ for an initial $\dot{M}_{\text {initial }}$ and an arbitrarily chosen $\alpha_{\text {initial }}$. We estimate the geometrical features of the model with the corresponding Shakura-Sunyaev disc solution. We then let the model evolve until it reaches a steady-state (one in which the surface density of the disc 
as a function of $r$ does not change in time) and compute $\dot{M}_{\text {improved }}$ from the stabilized model, which turns out to be different from the $\dot{M}_{\text {initial }}$. With this improved value for the mass transfer rate, we can now compute a new value for the $\alpha$-parameter $\alpha_{\text {improved }}$ as follows:

From conservation of mass we have in a steady-state $\dot{M}=2 \pi r \Sigma v_{\mathrm{r}}(\Sigma=\rho H$ is the surface density in the disc, and $v_{\mathrm{r}}$ the inward component of the fluid velocity). Since $\nu_{\text {eff }} \sim r v_{\mathrm{r}} 2 / 3$ we get $\nu_{\text {eff }} \sim \dot{M} / 2 \pi \Sigma$. Also $\alpha=\nu_{\text {eff }} / c_{\mathrm{s}} H$, which finally gives the proportionality

$\alpha \sim \frac{\dot{M}}{2 \pi \Sigma} \cdot \frac{1}{c_{\mathrm{s}} H}$

If we consider the values of $c_{\mathrm{s}}, H$ and $\Sigma$ to be fixed, we can find an improved $\alpha$ from the model expressed in the steady-state value of $\dot{M}$ :

$\alpha_{\text {improved }}=\alpha_{\text {initial }} \frac{\dot{M}_{\text {improved }}}{\dot{M}_{\text {initial }}}$.

The improved values of $\alpha$ and $\dot{M}$ can be used to construct a new disc geometry (based on a a new Shakura-Sunyaev solution) on which the simulation is repeated with the same values for the probabilities $S_{0}$ and $D$. Subsequent iterations give values of $\alpha$ which converge rapidly to a final value. For a set of free parameters $S_{0}=0.05$ and $D=0.1$, and for an initial arbitrary $\alpha_{\text {initial }}=0.1$ and $\dot{M}_{\text {initial }}=10^{16} \mathrm{~g} / \mathrm{s}$, the subsequent improved values $\alpha_{\text {improved }}$ converge to a final value of $\alpha_{\text {final }}=0.5$, while the mass accretion rate settles at a value of $2.5 \times 10^{16} \mathrm{~g} / \mathrm{s}$. This value corresponds to an accretion luminosity $L_{\text {acc }}$ equal to $1.7 \times 10^{-3} L_{\mathrm{Edd}}$, where $L_{\mathrm{Edd}}$ is the Eddington luminosity.

The simulation produces a final mass distribution in the disc (mass as a function of $r$ ) which exhibits fluctuations around a distribution which is steady in time and gives a total disc mass equal to a Shakura-Sunyaev mass distribution for $\alpha=\alpha_{\text {final }}$ and mass input rate equal to $\dot{M}_{\text {final }}$. However, not surprisingly, the $r$-dependence of this distribution deviates significantly from the corresponding Shakura-Sunyaev distribution for $r$-independent $\alpha$ (Fig. 4). This fact indicates that a completely selfconsistent representation of this disc would be one in which the $\alpha$ parameter would be $r$-dependent.

\subsection{Magnetic energy release}

For each time step of the simulation we keep a record of the total energy that is released due to reconnection of coronal magnetic loops (on one side of the disc). In this way, we construct a time series of the magnetic energy released in the part of the disc that is being modeled. For the emitted X-rays we consider two alternative cases: (1) The energy $E_{l}$ that is released at the time-step $t_{i}$ by a flaring loop $l$ is radiated in X-rays instantaneously, i.e. the emission-profile of every flaring loop is a $\delta$-function: $E_{l}(t)=E_{l} \cdot \delta\left(t-t_{i}\right)$. (2) The emission of a flaring loop increases instantaneously, but it is followed by an exponential decay (emission profile as a one-sided exponential):
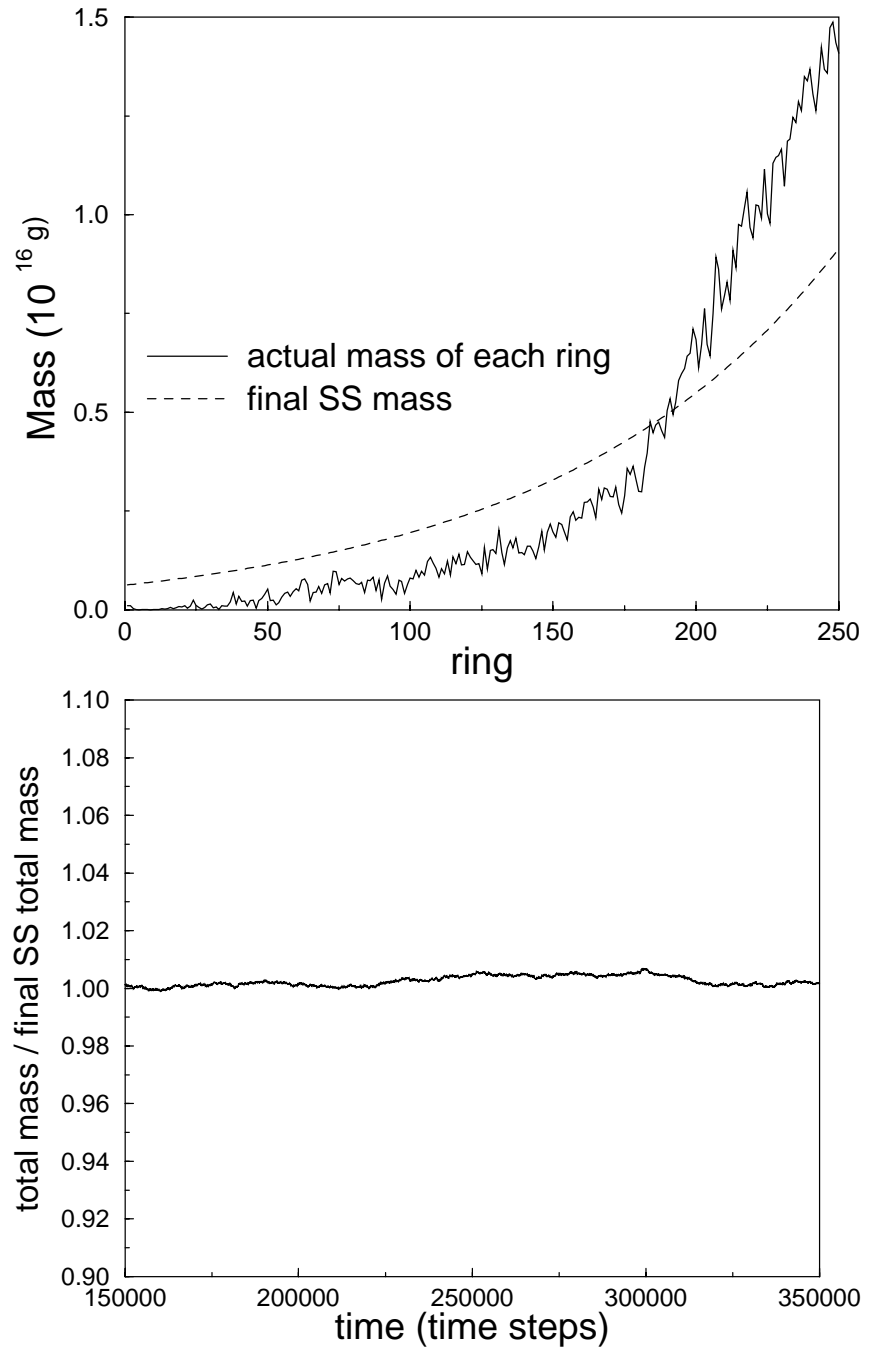

Fig. 4. Top: the mass of each ring after 350000 time steps $\left(D=0.1, S_{0}=0.05\right)$, plotted together with the ShakuraSunyaev mass distribution at the beginning of the final iteration. Bottom: the total disc mass as a function of time, plotted for the last 200000 time steps of our calculation.

$E_{l}(t)=E \cdot \mathrm{e}^{\left(-\frac{t-t_{i}}{\tau_{l}}\right)}$ (for $t \geq t_{i}$ ), where $E=E_{l} / \tau_{l}$, and the decay time $\tau_{l}$ is estimated as $\tau_{l}=0.1 L / c_{\mathrm{s}}$, where we have approximated the length of the loop by its footpoints distance $L$, and $c_{\mathrm{S}}$ is the sound-speed at the outer foot-point of the loop. In both cases, we keep track of the $\mathrm{X}$-ray emission as a time-series by counting the emissions from all flaring loops.

The corresponding time series are shown in Figs. 5 and 6, respectively. Clearly, an exponential emission profile causes temporally-extended structures to appear, whereas the time-series for instantaneous emission exhibits a more noise-like behaviour with, however, a more extended energy range. The amount of energy released per time step depends on the amount of flux present in the disc corona at any time step, that is to say on the flux filling factor and, consequently, on the probabilities $S_{0}$ and $D$.

In the case of the $\delta$-function emission-profile, the power spectrum of the time series is flat at low frequencies (white noise behaviour) and exhibits a turn-over to (weak) 

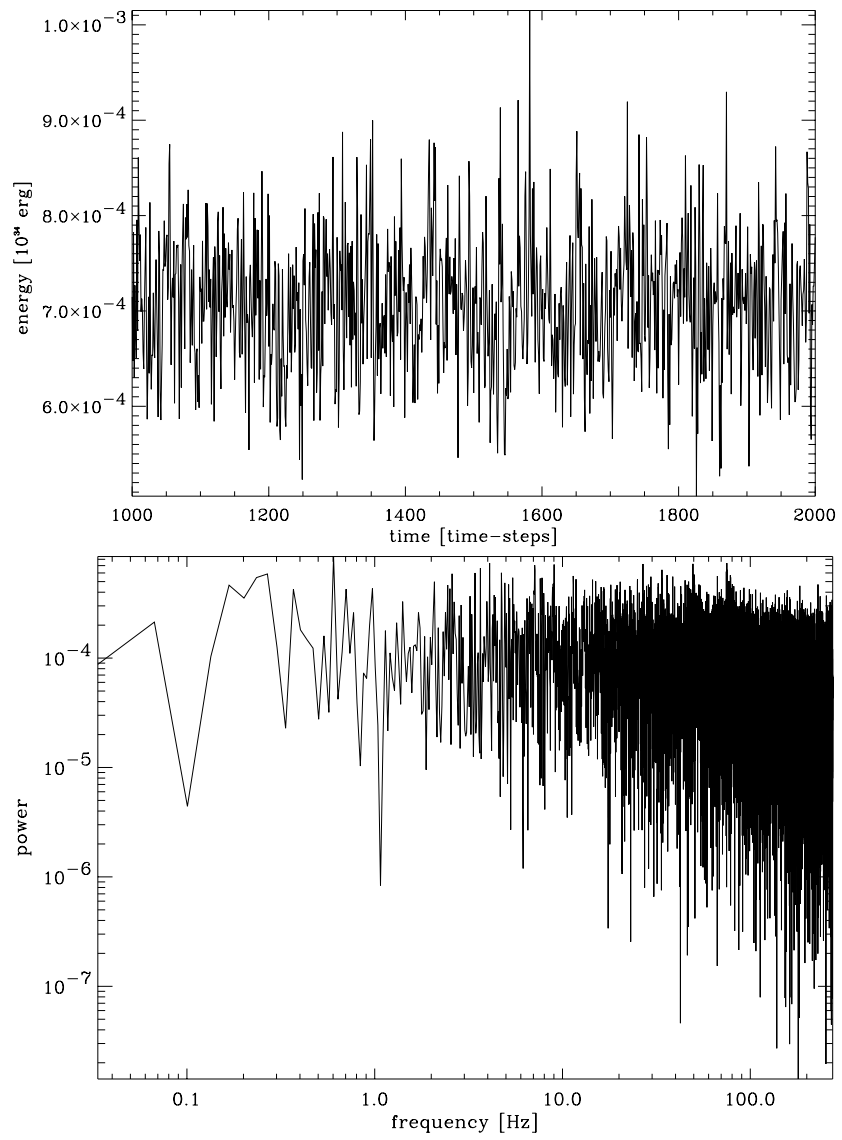

Fig. 5. Top: part of an energy release time series, using a $\delta$ function emission-profile for the flaring loops (instantaneous emission). Bottom: power spectrum of a time series of length 16384 time-steps, a part of which is shown in the top panel $\left(D=0.09, S_{0}=0.009\right)$. One time-step corresponds to $1.82 \times 10^{-3} \mathrm{~s}$.

power-law behaviour with slope of about -0.3 for large frequencies (above $100 \mathrm{~Hz}$; Fig. 5). For the exponential emission-profile, there is a clear power-law behaviour of the power-spectrum at frequencies already above roughly $5 \mathrm{~Hz}$, with slope -1.7 (Fig. 6). This is to be compared to the power-spectra of X-ray fluctuations of X-ray binaries, which exhibit power-law behaviour with indices between -1 and -1.7 (Makishima 1988). Our model with assumed exponential emission-profile thus reproduces a power-law with an index compatible with the observations.

Of course, for consistency with the Shakura-Sunyaev disc model which we simulate magnetically, most of the liberated energy must still be in blackbody radiation from the disc. For the case presented in Figs. 5 and $6(D=0.09$, $\left.S_{0}=0.009\right)$ the "magnetic" or X-ray luminosity fluctuates around $L_{\text {mag }}=3.8 \times 10^{33} \mathrm{erg} / \mathrm{s}$ (note that one timestep corresponds to $1.82 \times 10^{-3} \mathrm{~s}$ ). This should be compared to the disc accretion luminosity $0.5 G M \dot{M} / R_{*}=$ $1.6 \times 10^{36} \mathrm{erg} / \mathrm{s}$ for this case, or more precisely to the part released gravitationally between the outer and inner edge of the disk $L_{\mathrm{acc}}=6 \times 10^{35} \mathrm{erg} / \mathrm{s}$. The fraction of the accretion luminosity emitted via the magnetic channel is then only $L_{\text {mag }} / L_{\text {acc }} \sim 6.2 \times 10^{-3}$, which is consistent with the model assumptions.
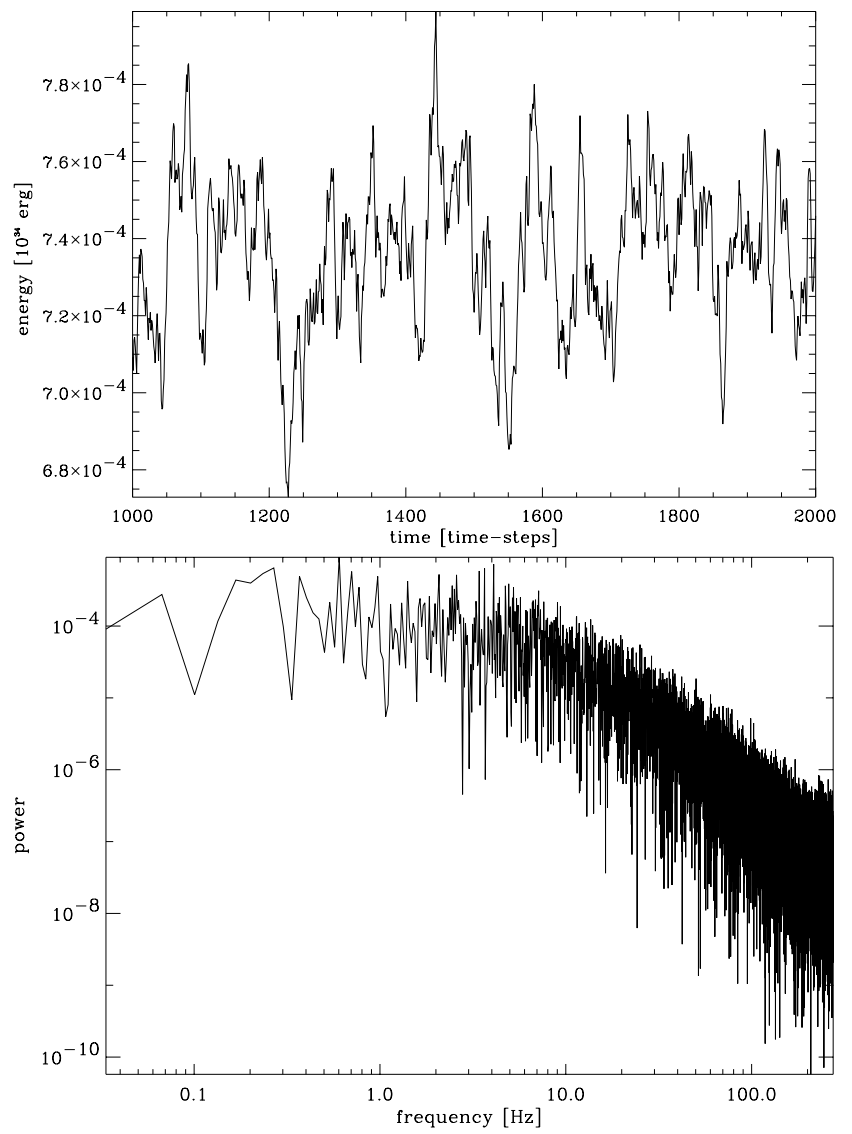

Fig. 6. Top: the energy release time series, using a one-sided exponential for the emission profiles of flaring loops. Bottom: power spectrum of a time series of length 16384 time-steps, a part of which is shown in the top-panel $\left(D=0.09, S_{0}=0.009\right)$. One time-step corresponds to $1.82 \times 10^{-3} \mathrm{~s}$.

\section{Summary and discussion}

We have presented a Cellular Automaton model to study the global features of accretion in a disc by the action of coronal magnetic loops, of the associated coronal magnetic activity, and of the magnetic energy release. The physical processes of spontaneous and stimulated appearance, and of diffusive disappearance of magnetic loops on the disc, are described in a probabilistic way, controlled by three free parameters (probabilities of diffusive disappearance, $D$, of spontaneous generation, $S_{0}$, and of stimulated generation $P$ ). We have investigated the role of two of these parameters: $D$ and $S_{0}$. The transfer of angular momentum, of mass, and the release of energy is based on straightforward approximations of the evolution of a single coronal loop, as specified by the initial locations of its foot-points and its magnetic flux. We simulate only one side of the disc and assume that the magnetic corona on both sides of the disc is, at any instant, symmetric with respect to the disc mid-plane. We have included the effect of the mirror corona in the rate of transport in our calculations.

In our simulations, we find that the action of magnetic loops does indeed take over the transport of angular momentum and mass $\dot{M}$ as forced initially by the 
$\alpha$-prescription in a Shakura-Sunyaev disk, and at a new steady-state characterized by $\alpha_{\text {final }}, \dot{M}_{\text {final }}$.

Our main result is that the distribution of coronal magnetic loops computed in the model is capable of sustaining an inward mass transfer at rates in the range of the values observed in accreting systems. Expressing the viscous action of the loops in the usual $\alpha$-parameter, we can model $\alpha \sim 0.5$ without any problem.

The model is well-behaved and stable for a range of combinations of the probabilities $S_{0}$ and $D$ controlling the magnetic activity. Our results suggest that the combination of probabilities $\left\{S_{0}, D\right\}$, for which the system stabilizes at specific $\dot{M}$ is not unique, but can be chosen from a certain range of values. We now discuss the qualitative and quantitative features of the magnetic energy release in our simulations, and how these can be understood from simple physical considerations.

\subsection{Energy and angular momentum budget}

The energy release due to magnetic reconnection of the loops results in a variability of the energy release time series with a power-spectrum which depends strongly on the assumed emission-profile of single flaring loops. Assuming instantaneous emission, a white noise power spectrum results below a relatively high turnover frequency, above which it becomes a power-law with exponent -0.3 . For exponential emission-profiles, there is a clear power-law behaviour of the power-spectrum with index -1.7 . The observed variations in X-ray luminosity have characteristics similar to the model with exponential emission profiles, namely power-spectra exhibiting power-laws with indices in the range from -1 to -1.7 . The magnitude of the magnetic luminosity in our model is small compared to the observed X-ray luminosities. Here we discuss how these results depend on the assumptions and parameter values in the present simulations.

The power-spectrum: CA as complexity models are able to show power-spectra for a number of different reasons: on the one hand, they may be in a self-organized critical (SOC) state, where a threshold-dependent process may cause chain-reactions (avalanches) which eventually spread over the whole grid, thereby introducing temporal correlations which are reflected in a non-flat powerspectrum. In these SOC models, the evolution rules are such that a certain global stress is always maintained over the grid, i.e. they restrict the freedom of choosing just any, though physically motivated, evolution rules for the $\mathrm{CA}$. On the other hand, any kind of (strong enough) temporal correlations which are introduced into the energy release process may lead to non-flat power-spectra, for instance in the form of temporally extended energy release. Finally, some kind of communication or triggering between individual loops is probably able to lead to nonflat power-spectra. Such triggering may be less restrictive than SOC-type evolution rules and allow more freedom in choosing the rules than in the SOC case.
The second possibility, extended emission-profiles, has been applied in this paper. It should be noted, however, that different emission profiles would yield different power spectra (see e.g. Isliker 1996, on the relation of emission profiles - power spectra). The power spectra are only weakly dependent on the elements of the model other than the emission profile.

The "non-thermal" energy release: We have already noted that consistency of our model with a ShakuraSunyaev disc requires the "non-thermal" (magnetic) luminosity to remain small in comparison to the accretion luminosity. While the magnitude of our magnetic luminosity does indeed satisfy this criterion, we also like to understand its magnitude, and, moreover, how it depends on the chosen parameter values.

The amount of energy that can be stored and, subsequently, released via the magnetic "channel" depends sensitively on the typical loop length. This follows from elementary considerations of energy and angular momentum conservation, and can be seen as follows.

Energy budget and optimizing the magnetic energy release: we check whether our prescription for the energy released in a magnetic flare in Sect. 2.2.3 satisfies conservation of energy.

When an element of mass $m_{\mathrm{f}}$ moves inward from one Keplerian orbit to another over a radial distance $\Delta r$ (which is the jump during one time step) the liberated energy - which can be stored in the magnetic field - follows from the virial theorem

$\Delta W=\Delta\left(0.5 W_{\text {grav }}\right)=\frac{G m_{\mathrm{f}} M \Delta r}{2 r^{2}}=\Delta J \Omega_{\mathrm{K}}(r)$,

where $W_{\text {grav }}=-G M m_{\mathrm{f}} / r$ is the gravitational energy of the mass element, $\Delta r$ is the absolute value of the radial step, $\Delta J$ the loss in angular momentum (in absolute value) per time step as before, and we have have used Eq. (18). The end result of Eq. (21) could, of course, have been written directly from conservation of energy, and it really is just a confirmation that our prescription is physically consistent. In the case of a loop, the inner foot-point is displaced inward and the outer foot-point outward. The energy liberated in this process then follows from Eq. (21) to be

$$
\begin{aligned}
\Delta W_{\text {loop }} & =\Delta J \Omega_{\mathrm{K}}\left(r_{1}\right)-\Delta J \Omega_{\mathrm{K}}\left(r_{2}\right)=\Delta J \Omega_{\mathrm{B}} \\
& =1.5 \Delta J \Omega_{\mathrm{K}}\left(r_{0}\right) \frac{r_{2}-r_{1}}{r_{0}}
\end{aligned}
$$

where, as before, $r_{0}=\left(r_{1}+r_{2}\right) / 2$. The energy which becomes available as stored free magnetic energy when an, initially radial, flux tube gets distorted during the buildup time of a flare, $\tau_{\mathrm{f}}$, is just $\tau_{\mathrm{f}} / t_{\mathrm{ts}}$ times the above value $\left(t_{\mathrm{ts}}\right.$ is the time step). This energy should be equal to the released magnetic energy. Using Eqs. (A.3) and (A.4) we can write this result as

$$
\begin{aligned}
1.5 \Omega_{\mathrm{K}} \tau_{\mathrm{f}} \frac{\Delta J}{t_{\mathrm{ts}}} \frac{r_{2}-r_{1}}{r_{0}} & =\dot{J}_{f} \frac{r_{2}-r_{1}}{r_{0}} \\
& =2 A_{2, \operatorname{phot}} \frac{B_{z}^{2}}{8 \pi} \frac{r_{2}}{r_{0}} f_{\mathrm{c}}\left(r_{2}-r_{1}\right),
\end{aligned}
$$


which, indeed, becomes identical to our estimate of the magnetic energy release in Sect. 2.2.3 once we put $r_{2} / r_{0}=$ 1 and $r_{2}-r_{1}=L$.

Finally, it follows directly from Eq. (22) that - for a fixed amount of angular momentum transferred - the release of energy increases proportionally to $\delta r=r_{2}-r_{1}$, the radial foot-point separation of the loop. Physically, this is obvious, as for a large loop a relatively small amount of the energy released at foot-point 1 is absorbed as orbital energy by matter at foot-point 2 when it receives the transferred amount of angular momentum.

Approximations: The model described here is only a first step in the study of the magnetic activity in accretion discs using Cellular Automata. It contains several simplifying assumptions:

$-\alpha$ is a constant: The geometry of our model is based on a Shakura-Sunyaev disc solution with $\alpha=$ const. However, our results indicate that this assumption is not valid if the dominant angular momentum transport mechanism is a distribution of coronal magnetic loops. This can be understood physically if we consider the fact that in a differentially rotating disc the time-scale of the action of the disc dynamo depends on the angular velocity and thus on the distance from the center. Since it is the magnetic loops produced by this dynamo that produce the viscosity described by $\alpha$, an $r$-dependence of $\alpha$ follows as a natural consequence. A refinement of the model using a geometry based on a Shakura-Sunyaev disc with $r$-dependent $\alpha$ is required to achieve a completely self-consistent final state;

- The absence of non-active loops;

- The foot-points of a loop remain fixed;

- The instantaneous versus exponential release of the flare energy in X-rays: when the flaring processes are known better, the energy release can be described more adequately;

- Steady-state: a refinement of the present model is required to explore modeling of strongly time-dependent accretion;

- Gas pressure dominated disc: for simplicity we have considered discs which are dominated by gas pressure and not by radiation pressure;

- Similarly, we have addressed discs around nonmagnetized objects only.

Further study is also required in order to determine the limits of the region on the $\left\{S_{0}, P, D\right\}$ plane outside of which the configuration of the model becomes unstable (the grid fills up or empties completely) with respect to magnetic activity and mass density. From an analysis of the connection of the $\mathrm{CA}$ with the MHD equations (see Isliker et al. 1998) we plan to gain more insight into the meaning of the free parameters of our model.

Acknowledgements. We would like to thank Drs. A. Anastasidis and D. Vassiliadis for critical reading of the initial article. The work of V. Pavlidou and L. Vlahos was supported by the program (PENED) of the General Secretary of Research and Technology of Greece. V. Pavlidou was also supported by the program SOCRATES of the European Community during her three month visit to the University of Utrecht, and gratefully acknowledges the hospitality at the Astronomical Institute in Utrecht. J. Kuijpers gratefully acknowledges financial support under the Erasmus Programme for collaboration and exchange of teachers with the University of Thessaloniki, and the hospitality at the Section of Astrophysics, Astronomy and Mechanics in Thessaloniki.

\section{Appendix A: Torque from magnetic loops}

Here we summarize the physics behind the estimates (4)-(8) for the torque exerted by a magnetic loop extending from an accretion disc into the ambient forcefree corona. A more extensive discussion can be found in Kuijpers (1995).

Angular momentum transport: Consider an individual coronal magnetic flux tube, anchored in the accretion disc at distances $r_{1}$ and $r_{2}\left(r_{1}<r_{2}\right)$, and, initially, oriented in the radial direction. We assume that the coronal part of the flux tube is force-free and remains so during its evolution (except during the short period of reconnection), and that force balance between Lorentz force and pressure force is established in a relatively thin layer just above the disc photosphere. Over this thin layer the flux tube expands and the "vertical" component of the magnetic field decreases from its photospheric value $B_{z \text {,phot }}$ to its coronal value $B_{z \text {,cor }}=f_{\mathrm{c}} B_{z \text {,phot }}$, where $f_{\mathrm{c}}$ is the effective local surface filling factor defined before.

We make a distinction between flux tubes which are distorted appreciably by the flow at their foot-points and, consequently, reconnect in a magnetic flare, and flux tubes which are sufficiently compact to ultimately withstand the flow at the foot-points.

In a long flux tube with finite cross-section, Keplerian motion at the foot-points of the individual field lines within the tube shears and twists the internal magnetic field distribution. Both shear and twist change the current distribution in the coronal part of the tube. The fluid shear builds up an azimuthal (toroidal) magnetic field component out of an initially meridional (poloidal) field according to

$B_{\phi}=\frac{B_{z} \Omega_{\mathrm{B}} r t}{\delta r}$

where $\delta r \equiv r_{2}-r_{1} \ll r_{1}$ is the radial foot-point separation of the loop, and

$\Omega_{\mathrm{B}} \equiv\left|\Omega_{\mathrm{K}}\left(r_{1}\right)-\Omega_{\mathrm{K}}\left(r_{2}\right)\right|$

is the Keplerian beat frequency. Equation (A.1) is an approximation of the evolution of a linear force-free arcade to within $30 \%$ (Burm \& Kuperus 1988). The free magnetic energy of the flux tube increases, and, therefore, it expands upward into the corona. We assume that the flux tube reconnects with the overlying coronal field structure and produces a magnetic flare explosion as soon as the 
coronal values satisfy $B_{\phi}=B_{z}$ (Aly 1985; Kuijpers 1992). This occurs after a period

$\tau_{\mathrm{f}}=\frac{\delta r}{\Omega_{\mathrm{B}} r}=\frac{2}{3 \Omega_{\mathrm{K}}(r)}$,

which reproduces our Eq. (4). Of course, the tube also becomes twisted, at the same time as it is sheared, but much less. In our simple treatment we neglect the effects of twisting. The average rate of transport of angular momentum through a flaring loop can now be calculated from a straightforward integration of the Maxwell stresses across the (coronal) foot-point area $A_{2}$

$$
\begin{aligned}
\dot{J}_{\mathrm{f}} & \approx \frac{2 A_{2} B_{z 2} r_{2}}{4 \pi \tau_{\mathrm{f}}} \int_{0}^{\tau_{\mathrm{f}}} \frac{B_{z 2} \Omega_{\mathrm{B}} r_{2} t}{r_{2}-r_{1}} \mathrm{~d} t=\frac{A_{2} B_{z 2}^{2} r_{2}}{4 \pi} \\
& =2 A_{2} \rho c_{\mathrm{s}}^{2} r_{2} f_{\mathrm{c}}^{2},
\end{aligned}
$$

where the integration is over foot-point 2 and we have assumed that the loop is a closed flux tube which extends on both the upper and lower side of the disc in an antisymmetric fashion. The result (A.4) is our earlier Eq. (5).

In a sufficiently small flux tube the difference in angular momentum of the gas at both foot-points is so small that the loop magnetic field can transport the excess within a flaring time $\tau_{\mathrm{f}}$. Building up of an azimuthal field component satisfying (A.1) will then be halted when the loop reaches rigid rotation: $\Omega\left(r_{1}\right)=\Omega\left(r_{2}\right)=\Omega_{\mathrm{K}}\left(r_{0}\right)$ where $r_{0}=\left(r_{1}+r_{2}\right) / 2$. An estimate for the transfer time $\tau_{\mathrm{s}}$ is obtained from equating the deficit angular momentum at foot-point 2 with respect to that at a rotation rate $\Omega_{\mathrm{K}}\left(r_{0}\right)$ to the amount transported into foot-point 2 during a time $\tau_{\mathrm{s}}$ (use the first part of (A.4)):

$1.5 A_{\mathrm{p} 2} H_{2} \rho_{2} r_{2}\left(r_{2}-r_{1}\right) \Omega_{\mathrm{K} 2}=3 A_{2} r_{2} B_{z 2}^{2} \Omega_{\mathrm{K} 2} \tau_{\mathrm{s}}^{2}(8 \pi)^{-1}$,

where the cross-section of the flux tube at the level of the photosphere $A_{2, \text { phot }}$ relates to that at the corona as $A_{2, \text { phot }}=f_{\mathrm{c}} A_{2}$. For small loops, $f_{\mathrm{c}}$ will not be too different from unity, and it follows that

$\tau_{\mathrm{s}}=\left(\frac{r_{2}-r_{1}}{2 c_{\mathrm{s}} \Omega_{\mathrm{K}}}\right)^{0.5}=\frac{1}{\Omega_{\mathrm{K}}}\left(\frac{r_{2}-r_{1}}{2 H}\right)^{0.5}$,

which reproduces (7). Finally, the average rate of transport of angular momentum into footpoint 2 for a small loop during a time $\tau_{\mathrm{s}}$ is

$\dot{J}_{\mathrm{s}} \approx A_{2} 3 \rho c_{\mathrm{s}}^{2} r_{2}\left(\frac{r_{2}-r_{1}}{2 H}\right)^{0.5}=\left(\frac{9\left(r_{2}-r_{1}\right)_{\mathrm{s}}}{8 H}\right)^{0.5} \dot{J}_{\mathrm{f}}$

which reproduces (8).

\section{References}

Abramowicz, M. A., Lanza, A., Spiegel, E. A., \& Szuskiewicz, E. 1992, Nature, 356, 41

Aly, J. J. 1985, A\&A, 143, 19

Aly, J. J., \& Kuijpers, J. 1990, A\&A, 227, 473

Balbus S. A., \& Hawley J. F. 1991, ApJ, 376, 214

Blandford, R. D., \& Payne, D. G. 1982, MNRAS, 199, 883
Bracco, A., Provenzale, A., Spiegel, E. A., \& Yecko, P. 1999, to appear in Proc. of the Conf. on Quasars \& Accretion Disks, ed. M. Abramowicz (Cambridge Univ. Press)

Burm, H., \& Kuperus, M. 1988, A\&A, 192, 165

Contopoulos, J., \& Lovelace, R. V. E. 1994, ApJ, 429, 139

Coroniti, F. V. 1981, ApJ, 244, 587

de Vries, M., \& Kuijpers, J. 1992, A\&A, 266, 77

Fender, R. P., \& Hendry, M. A. 2000, MNRAS, 317, 1

Field, G. B., \& Rogers, R. D. 1993, ApJ, 403, 94

Frank, J., King, A., \& Raine, D. 1992, Accretion Power in Astrophysics (Cambridge Univ. Press), Chapt. 5.6 - 5.8

Galeev, A. A., Rosner, R., \& Vaiana, G. S. 1979, ApJ, 229, 318

Hawley, J. F., \& Balbus, S. A. 1999, in Numerical Astrophysics, ed. S. M. Miyama, K. Tomisaka, \& T. Hanawa (Kluwer, Acad. Publ., Dordrecht), 187

Heyvaerts, J., \& Priest, E. R. 1989, A\&A, 216, 230

Horne, K. D. 1994, in Theory of Accretion Disks - 2, ed. J. W. Duschl, J. Frank, F. Meyer, E. Meyer-Hofmeister, \& W. M. Tscharnuter (Kluwer Acad. Publ., Dordrecht), 77

Howard, R. F. 1996, ARA\&A, 34, 75

Isliker, H. 1996, A\&A, 310, 672

Isliker, H., Anastasiadis, A., Vassiliadis, D., \& Vlahos, L. 1998, A\&A, 335, 1085

Kuijpers, J. 1992, in The Sun: A Laboratory for Astrophysics, ed. J. T. Schmelz, \& J. C. Brown, Proc. NATO ASI (Kluwer Acad. Publ.), 535

Kuijpers, J. 1995, Flares in Accretion Discs., in Coronal Magnetic Energy Releases, ed. A. O. Benz, \& A. Krüger (Springer-Verlag), 135

Lin, D. N. C., \& Papaloizou, J. C. B. 1996, ARA\&A, 34, 703

Lubow, S. H., \& Pringle, J. E. 1993, ApJ, 409, 360

Lynden-Bell, D. 1969, Nature, 223, 690

Makishima, K. 1988, in Physics of Neutron Stars and Black Holes, ed. Y. Tanaka (Universal Academy Press, Tokyo), 175

Mestel, L. 1999, Stellar Magnetism (Oxford Univ. Press Oxford, UK)

Michel, F. C. 1984, ApJ, 279, 807

Miller, K. A., \& Stone, J. M. 1999, in Numerical Astrophysics, ed. S. M. Miyama, K. Tomisaka, \& T. Hanawa (Kluwer, Acad. Publ., Dordrecht), 237

Mineshige, S., Ouchi, N. B., \& Nishimori, H. 1994, PASJ, 46, 97

Nauta, M. D., Kuijpers, J., \& Zimmerman, J. T. F. 2000, A\&A, submitted

Nelson, R. F., \& Spencer, R. E. 1988, MNRAS, 234, 1105

Papaloizou, J. C. B., \& Lin, D. N. C. 1995, ARA\&A, 33, 505

Papaloizou, H. C. B., \& Pringle, J. E. 1977, MNRAS, 181, 441

Pozdnyakov, L. A., Sobol, I. M., \& Sunyaev, R. A. 1983, Sov. Sci. Rev. E, 2, 189

Pounds, K. A., \& McHardy, I. M. 1988, in Physics in Neutron Stars and Black Holes, ed. Y. Tanaka, Tokyo, Japan, 285

Pringle, J. 1981 ARA\&A, 19, 137

Rozyczka, M., \& Spruit, H. C. 1993, ApJ, 417, 677

Schramkowski, G. P., \& Torkelsson, U. 1996, A\&AR, 7, 55

Seiden, P. E., \& Wentzel, D. G. 1996, ApJ, 460, 522

Shakura, N. I., \& Sunyaev, R. A. 1973, A\&A, 24, 337

Stehle, R., \& Spruit, H. C. 1999, submitted to MNRAS

Tagger, M., Henriksen, R., Pellat, R., \& Sygnet J. F. 1990, ApJ, 353, 654

Tout, C. A., \& Pringle, J. E. 1992, MNRAS, 259, 604

Weiss, N. 1997, in Advances in the Physics of Sunspots, ed. B. Schneider, J. C. del Toro Iniesta, \& M. Vazquez, Astronomical Society of the Pacific, 21

Wentzel, D. G., \& Seiden P. E. 1992, ApJ, 390, 208 